DOI: $10.3901 / J M E .2020 .15 .118$

\title{
折纸结构驱动技术的研究进展
}

\author{
胡 楠 1,2 陈花玲 1,2 \\ (1. 西安交通大学机械工程学院 西安 710049; \\ 2. 西安交通大学机械强度与振动国家重点实验室 西安 710049)
}

\begin{abstract}
摘要: 折纸结构因具有强大的折展能力以及在应用中制造装配简单等优势, 为许多复杂工程问题的解决提供了新思路, 因此 吸引了航天、医疗、机器人等领域的不少学者们对其开展研究。在折纸技术研究领域, 折纸结构的驱动技术是其中一个关键 技术。按照驱动原理的不同, 将折纸驱动技术分为气动驱动、机械式驱动、活性材料驱动三大类, 然后分别对其国内外研究 现状进行归纳和分析, 并对各种驱动方法的优缺点、适用场合等进行对比, 最后重点指出了基于活性材料的折纸结构驱动技 术研究面临的问题以及未来的发展趋势。
\end{abstract}

关键词: 折纸; 驱动; 气动; 机械式结构; 活性材料

中图分类号: TH112

\section{Progress in Actuating Technology of Origami Structure}

\author{
HU Nan ${ }^{1,2}$ CHEN Hualing ${ }^{1,2}$
}

(1. School of Mechanical Engineering, Xi’an Jiaotong University, Xi'an 710049;

2. State Key Laboratory of Mechanical Structural Strength and Vibration,

Xi'an Jiaotong University, Xi’an 710049)

\begin{abstract}
Origami structure has many advantages, such as strong folding ability and simple manufacturing and assembly in application, and it provides many new ideas for solving many complex engineering problems. Therefore, origami attracts many scholars in aerospace, medical, robotics and other fields to research. In the research of origami, the actuation technology of origami structure is one of the important technologies. According to the difference in actuation principle, the origami actuation technology is divided into three categories, and they are pneumatic actuating, mechanical structure actuating and active material actuating. The current research situation of origami actuation technology is summarized and analyzed, and the advantages, disadvantages and suitable occasions of different methods are compared. Finally, the problems faced by the research of origami structure actuation technology based on active materials and the development trends in the future are put forward.
\end{abstract}

Key words: origami; actuation; pneumatic; mechanical structure; active material

\section{0 前言}

折纸作为一种传统的手工艺术形式, 它可以把 未剪裁的二维纸张折叠成具有艺术性的形状多样的 三维艺术品。折纸技术最早始于中国, 在中国有了造 纸技术之后折纸技术就开始逐渐发展, 唐代时流传至 日本, 之后逐渐传至欧美各国。折纸的最初目的不是 在于实用价值, 而是在于娱乐和艺术价值, 直到 20 世纪 70 年代中期, 数学家发现理论上可以用传统的 折纸术创造出无数的形状 ${ }^{[1]}$, 且随着计算机技术的崛

20190827 收到初稿, 20191217 收到修改稿
起, 折纸的设计也可以从传统的依靠设计师的经验逐 渐变为由计算机辅助技术实现 ${ }^{[2]}$ 。于是, 人们逐渐开 始探索将折纸技术用于解决各种复杂工程问题, 折纸 的价值也从单纯的艺术领域拓展到工程领域。

折纸在折叠过程中每一个纸片上任意两个点之间 的表面距离保持不变，且纸片之间不发生拉伸、撕裂 和互相侵入, 其变形的本质是折痕处的弯曲变形 ${ }^{[3]}$ 。与 其他结构相比, 折纸具有很强的折叠和展开能力, 这 使得基于折纸的结构变得更加紧凑; 此外, 折纸结构 的制造和装配也非常简单，它不需要用于传递运动的 运动副, 从而可减少部件数量, 而且装配方式也更加 灵活 ${ }^{[4]}$ 。这些优势使得折纸结构在诸多领域具有巨大的 应用前景, 从而吸引了大量学者开展相关研究。例如, 
利用折纸结构具有的折叠和展开功能, 可设计出满 足航天工程要求的结构紧凑和重量轻的空间太阳能 帆板 ${ }^{[5]}$ 。由于折纸运动功能的实现与尺度无关, 因此 在微小型结构中同样可以应用, 这也使得它具有了 在医疗卫生行业当中应用的潜力 ${ }^{[6-9]}$, 比如人造血管 支架 ${ }^{[10]}$ 和体内药物抓取释放结构等 ${ }^{[11]}$ 。此外, 近年 来折纸结构在机器人 ${ }^{[12-15]}$ 、可折叠机翼飞机 ${ }^{[16-18]}$ 、 碰撞能量吸收系统 ${ }^{[19-22]}$ 、可折叠购物袋 ${ }^{[23]}$ 、智能电 子元器件 ${ }^{[24-27]}$ 、光学设备 ${ }^{[28-29]} 、$ 帐篻等遮蔽物 ${ }^{[30-32]}$ 、 超材料 ${ }^{[33-34]}$ 等领域的探索均有报导。图 1 为折纸结构 在部分领域应用的案例, 由此可见, 通过某种构思将 折纸的思想运用到折叠结构中可以实现结构的折叠与 展开, 对于多个领域的进步和发展都具有重要的意义。

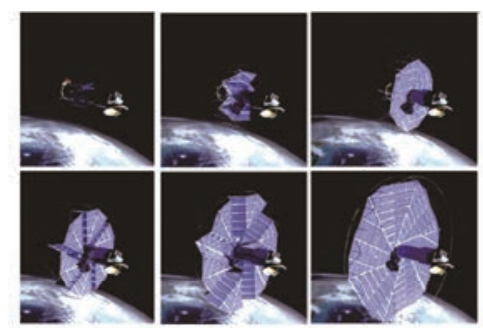

(a) 空间太阳能帆板 ${ }^{[5]}$

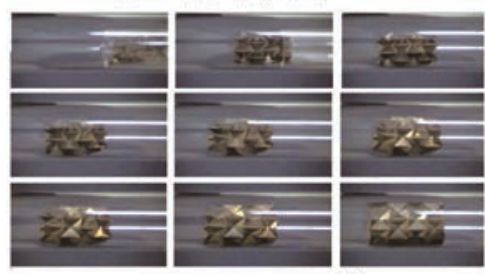

(b) 人造血管支架 ${ }^{[10]}$

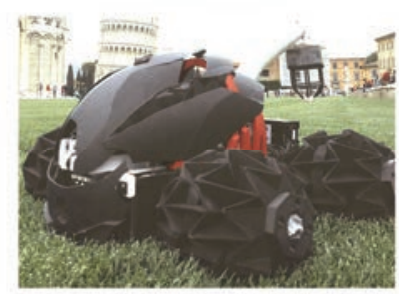

(c) 可变直径车轮爬行机器人 [15]

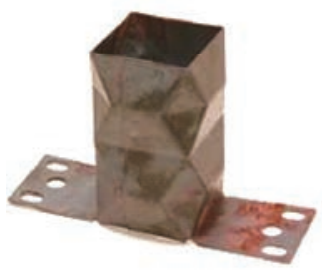

(d) 汽车碰撞能量吸收盒 ${ }^{[21]}$

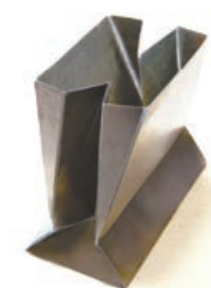

(e) 可折叠购物袋 ${ }^{[23]}$
图 1 折纸结构在多个领域中的应用

然而, 总体来看, 目前折纸技术在工程中的应 用还处于探索阶段, 若要将其更好地用于解决实际 工程问题还面临很多挑战, 这其中除了需要深入研 究折纸理论、开发新型折纸结构之外, 一个重要的 研究领域就是折纸结构折展的驱动技术。显然, 对 于可动折纸, 驱动方法将会直接决定预期运动功能
的实现, 若驱动问题不能得到有效解决, 那么设计 巧妙的折纸也将失去其实际应用价值, 因此, 折纸 结构的驱动技术是折纸领域研究的一个关键技术。

按照驱动原理的不同, 现有的折纸驱动方法主要 有气动驱动、机械式驱动、活性材料驱动(包括热活性 材料、化学活性材料、电活性材料、磁活性材料、光 活性材料等)。为了促进折纸技术的深入发展, 本文将 以折纸结构的驱动技术为出发点, 分别对目前几类驱 动技术研究现状展开讨论, 分析各种驱动方法的优势 和所面临的问题以及其适用的驱动场合, 最后对未来 折纸结构活性材料驱动技术的发展趋势进行展望。

\section{1 气动驱动在折纸结构中的应用}

气动驱动主要用于一些封闭管状折纸结构的驱 动, 一般通过充气和抽气来控制折纸的展开和折叠。 例如, 哈佛大学 MARTINEZ 以折纸为模板, 通过 浇铸复合材料 Ecoflex 制备了一种充气式管状折纸, 如图 2 所示 ${ }^{[35]}$, 他们根据需要在不同区域对折纸进行 粘接, 当对管状折纸充气时, 被粘接一侧因运动受限 而无法伸展, 进而导致两侧伸展程度不一致产生弯曲 变形, 经过人为设计可以沿一定的路径折展, 并且能 够托起自重 120 倍的重物 ${ }^{[35]}$ 。方虹斌等人设计了一种 球状 Waterbomb 折纸结构, 如图 3 所示 ${ }^{[36]}$, 他们将一 个气球放置在折纸内, 并在气球的两端连接刚性平板, 其中一端的平板固定，另一端可以移动。当对气球充 气时, 气球膨胀并带动端部平板移动, 进而使折纸球 展开; 同理, 对气球抽气时可以实现折纸球的收缩 ${ }^{[36]}$ 。

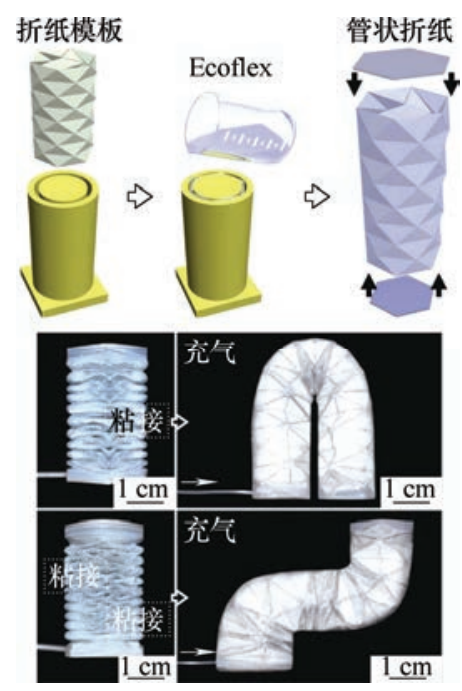

图 2 气动驱动的折纸管

由此可知, 气动驱动可以对具有封闭腔体的折 纸结构进行驱动, 实现伸展和折叠运动, 还可以通 过一些特殊的结构设计实现弯曲等运动功能。气动 
驱动的优势是可以产生很大的驱动力, 如图 4 所示, 其局限性是气动驱动需要气原、气阀等其他辅助设 备, 对于难以形成封闭腔体的折纸或者不具备气原 设备的特殊场合, 气动驱动就不能发挥作用 ${ }^{[37]}$ 。
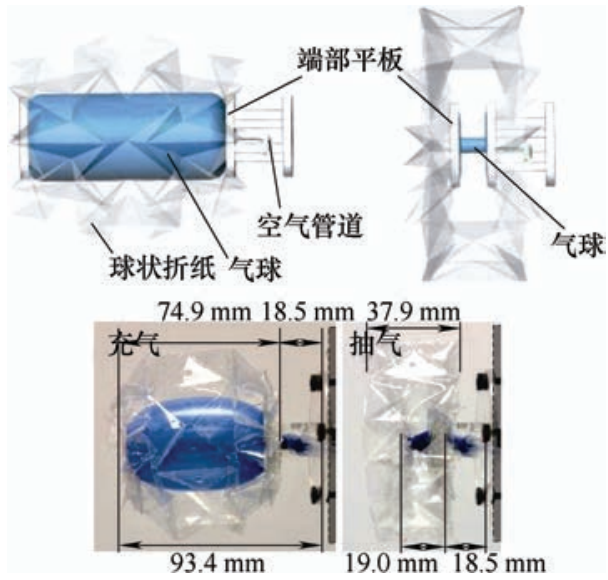

图 3 气动驱动的折纸球

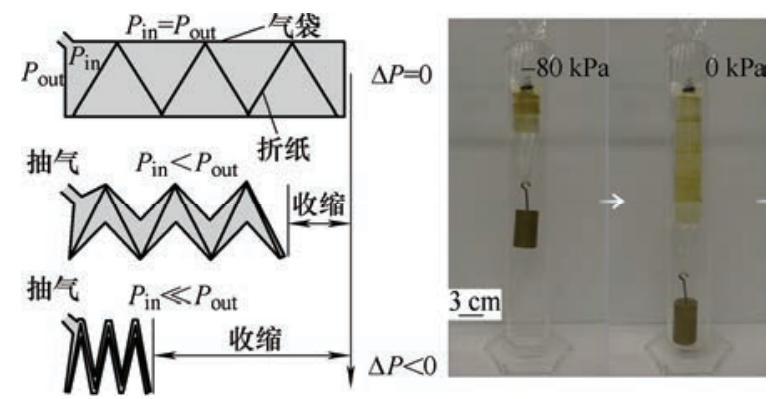

图 4 气动驱动的可伸缩人工肌肉

\section{2 机械式驱动在折纸结构中的应用}

机械式驱动一般以电动机为动力源, 通过绳索、 齿轮等机械结构作为传动工具对折纸进行驱动, 其 中, 最常用的驱动结构是电动机带动绳索进行驱动。 例如, 首尔大学 $\mathrm{KIM}$ 等 ${ }^{[38]}$ 通过电动机带动绳索牵拉 纸板, 成功地对 Sarrus 折纸结构进行了驱动, 如图 5 所示, 并以特殊的结构设计实现了完全展开后的 自锁功能, 最终将此技术应用于无人机智能操作臂。 伦敦大学 ZHANG 等 ${ }^{[39]}$ 将三根绳索置于管状折纸内, 通过电动机拉动 3 根绳索实现了折纸管的收缩运 动, 并且通过控制 3 根绳索的拉力差实现弯曲运动。 类似的, 凯斯西储大学 JEDNG 等 ${ }^{[40]}$ 也通过伺服电 动机和绳索控制管状折纸设计了一种抓取器, 如图 6 所示, 该结构不仅实现了 “手臂” 的收缩和弯曲运动, 还通过绳索控制了底端三个 “手指” 的弯曲方向, 从 而实现了抓取功能。首尔大学 LEE 采用 Waterbomb 折纸结构设计了一种可变车轮直径的爬行机器人, 如图 7 所示, 该车轮形状的改变依靠滑轮和一个具 有多层结构(包含弹簧、特氟隆管、拉线)的特殊绳
索实现, 绳索的一端固定在车轮外侧轮毂上。当拉 线处于松弛状态时, 车轮保持椭球形状; 当电动机 带动滑轮转动拉紧拉线时, 车轮的轴向收缩、径向 膨胀, 从而实现了车轮直径的变化 ${ }^{[15]}$ 。

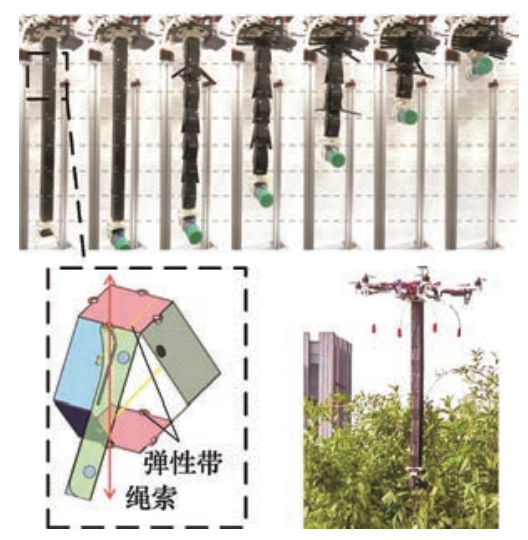

图 5 绳索驱动的智能无人机操作臂

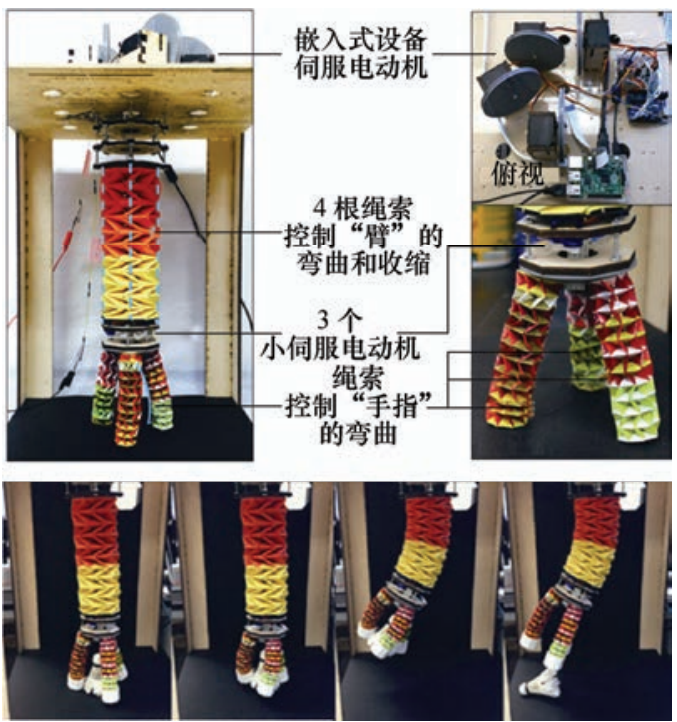

图 6 绳索驱动的折纸抓手

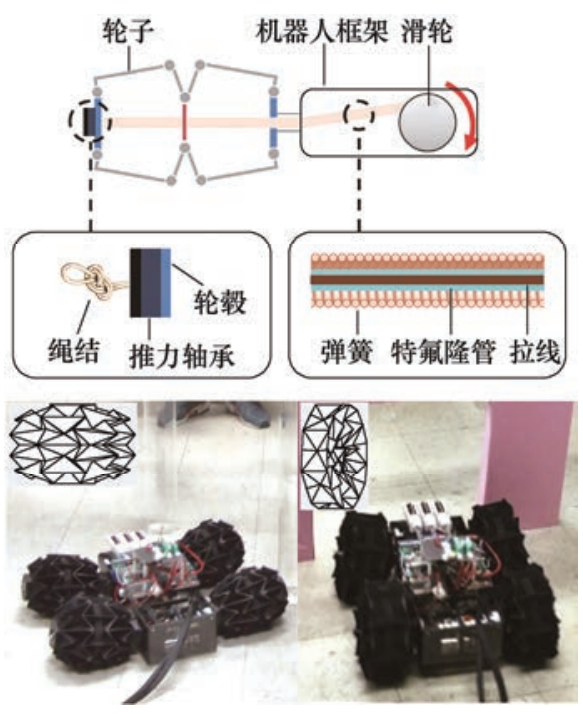

图 7 绳索驱动的可变车轮直径爬行机器人 
电动机带动齿轮的驱动方式在折纸中也有探 索。例如, 美国东北大学 KAMRAVA 等 ${ }^{[41]}$ 根据条 状折纸设计了一种模仿人手指的抓手, 如图 8 所示 $(1 \mathrm{inch}=2.54 \mathrm{~cm})$, 该折纸在折痕处增加了铰链, 而 铰链又与齿轮连接, 再通过电动机驱动齿轮转动实 现单根 “手指” 的运动, 并在此基础上对五根 “手 指” 进行设计, 使之能够协作工作, 可以模仿人手 完成一系列复杂动作。
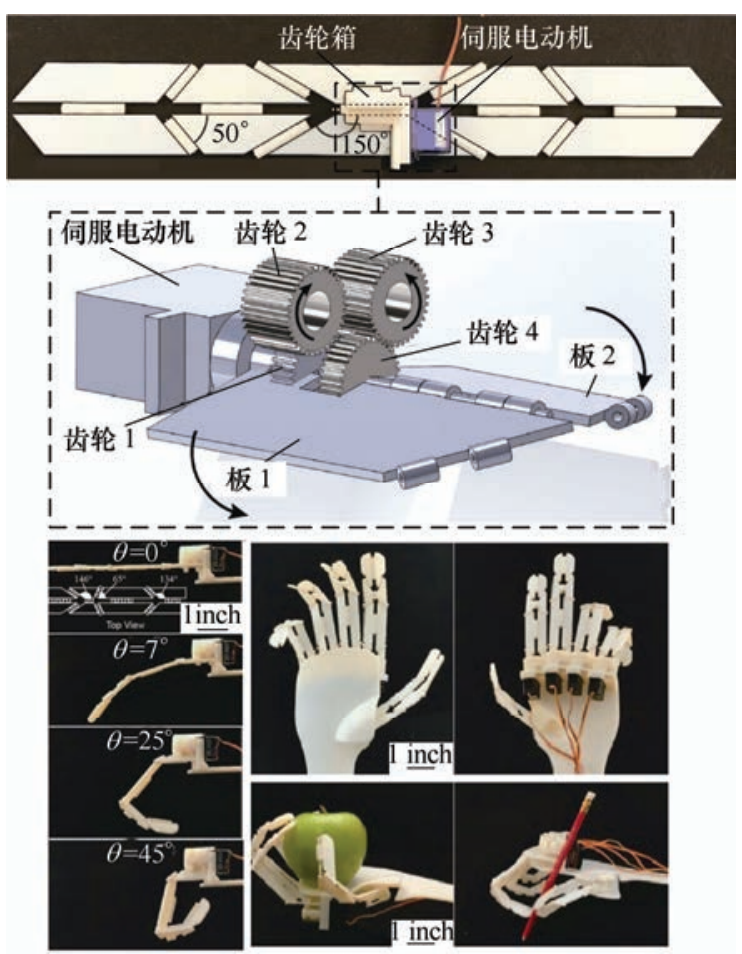

图 8 电动机-齿轮-铰链驱动的条状折纸及其应用

机械式驱动方法是最经典的机械驱动方式, 它能 够对结构进行较为精准的控制以完成预期的运动目 标, 而且在驱动力和运动范围方面也具有明显优势, 对于较大尺寸的刚性折纸结构的驱动是一种不可替 代的最佳选择。然而, 机械式驱动方法需要电动机、 绳索、齿轮、弹簧等传统机械零部件, 对于轻小型折 纸结构必定会增加结构的复杂程度和重量。此外, 由 于绳索具有只能受拉不能受压的特性, 因此仅能完成 折纸的收缩运动, 而伸展运动还需依靠弹簧等其他元 件。由此可见, 机械式驱动方法一般适用于大型结构, 若用于小型结构则会丧失折纸本身的独特优势。

\section{3 活性材料驱动在折纸结构中的应用}

活性材料是一类可以在外界激励下性能发生变 化的材料, 它可以将其他形式能量转换为机械能, 因 此, 活性材料可以作为驱动材料, 也为解决折纸的驱 动问题提供了一种新途径。现有的活性材料根据工作
原理的不同可分为热活性材料、电活性材料、化学活 性材料、磁活性材料、光活性材料等, 国内外也有大 量文献报导通过各类活性材料驱动折纸的研究。

\section{1 热活性材料驱动}

热活性材料一般在温度变化时其形状也发生变 化, 其热能的获得可以由热传导、焦耳加热、对流、 辐射等途径, 热能的来源一般有温度场、电流、光 照、化学反应等, 但是其发生变形的本质是受热变 形。在空间上, 热活性材料可以采用全局供热, 也 可以是局部供热; 在时间上, 可以同时供热，也可 以按照一定时间序列供热。热活性材料种类众多, 能用于折纸驱动的有形状记忆合金(Shape memory alloy, SMA)、形状记忆聚合物 (Shape memory polymer, SMP)和各类热缩型高分子聚合物。

形状记忆材料因具有较大的应力、应变范围, 目 前成为活性材料中应用最多的一种驱动材料, 在折纸 结构驱动中的应用也不例外。最典型的驱动方式是将 形状记忆材料放置于折纸的折痕处, 例如, 哈佛大学 TOLLEY ${ }^{[42]}$ 等将具有弯曲变形记忆的 SMP 置于折纸的 折痕处, 并通过设计合适的纸片厚度和 SMP 材料参数 对每一处折痕的最大转角进行控制, 在均匀温度场中 实现了多面体折纸由展开到折叠的变形, 如图 9 所示。 在此基础上, FELTON 等 ${ }^{[43]}$ 又将 SMP 材料、电热丝与 折纸板进行集成，在外界电源控制下分时、局部加热， 实现了纸板在非均匀温度场中折叠, 且不同折痕的转 动角度和运动先后顺序均可控制, 最终研制出了一种 爬行机器人, 如图 10 所示。与 SMP 类似, SMA 也可 以用作折纸的驱动。例如, PAIK 等 ${ }^{[44]}$ 将 SMA 置于折 纸的折痕, 通过加热使得二维纸片变成桌子、风车 等形状的三维造型, 如图 11 所示。

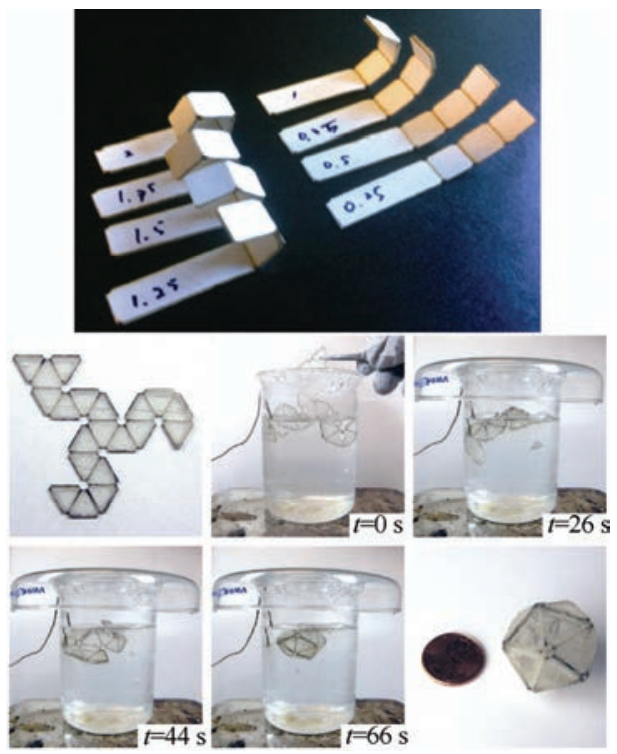

图 9 均匀温度场中 SMP 驱动的折纸 


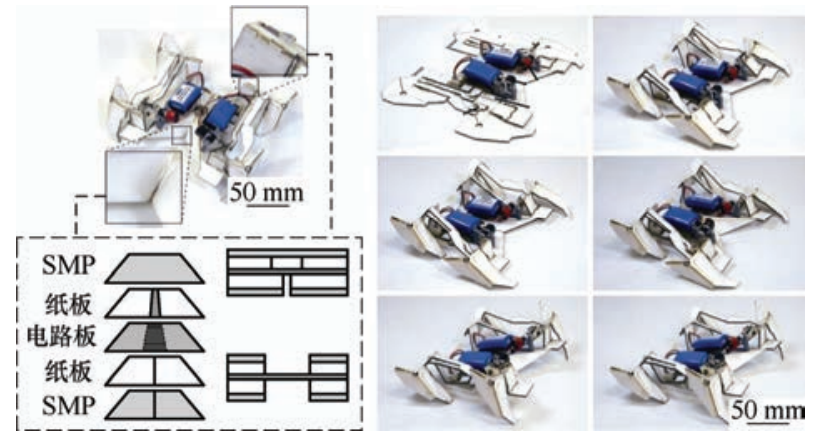

图 10 SMP 驱动的折纸爬行机器人
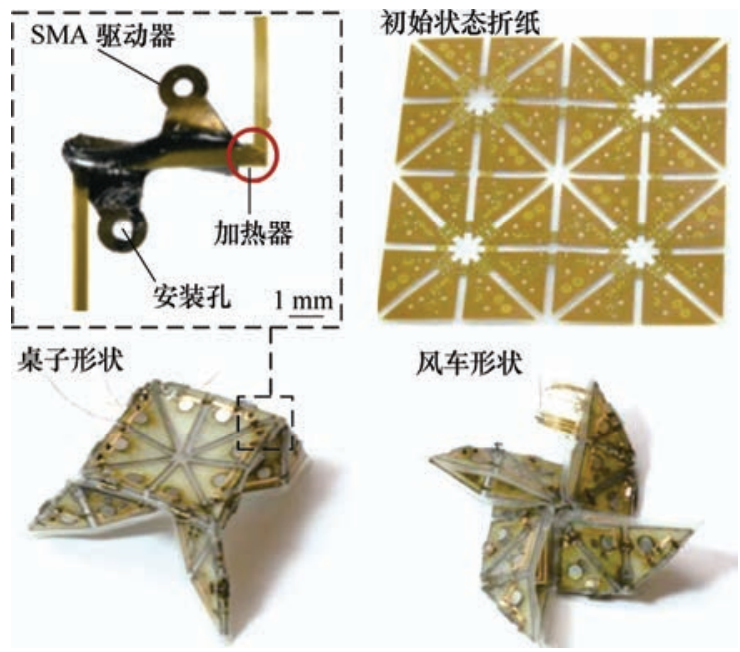

图 11 SMA 驱动的可变造型折纸

形状记忆材料除了用于折痕处对折纸进行驱动 外, 还可以将其用于折纸外部进行驱动。例如, 美国 ONAL 等 ${ }^{[45]}$ 将 SMA 弹簧附着在分段管状折纸的外部 制成一种爬行器, 如图 12 所示, 然后控制每一段折纸 上的 SMA 弹簧的拉伸和收缩, 依靠爬行器的 “腿” 与 地面摩擦向前爬行, 从而达到模仿虾蚂运动的效果。
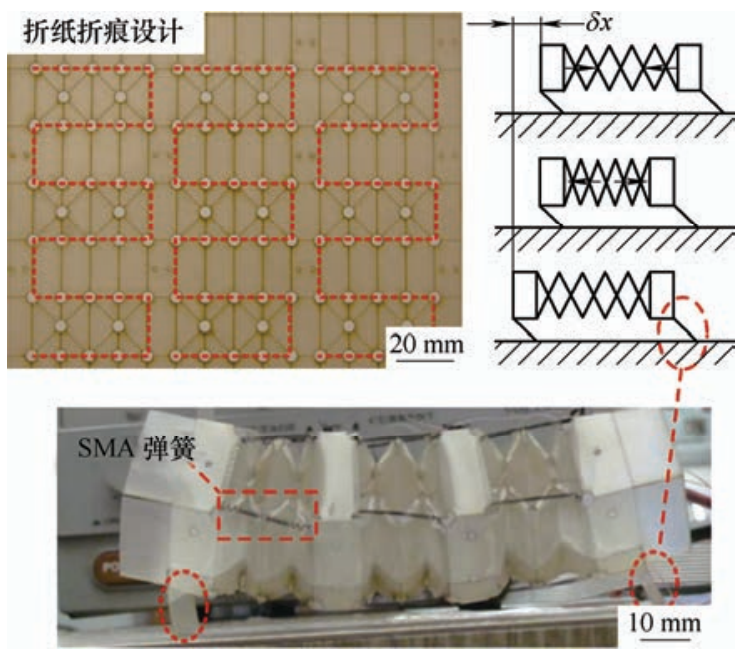

图 12 SMA 弹簧驱动的管状折纸爬行器

热缩型高分子聚合物可以预热收缩, 这种变形 也可以通过一定的结构设计间接地转化成弯曲变
形, 从而用来驱动折纸。例如, 麻省理工的计算机 科学和人工智能实验室 MIYASHITA 等 ${ }^{[46]}$ 采用热缩 型聚氯乙烯(Polyvinyl chloride, PVC)和厚纸板夹层结 构设计了一种折叠铰链, 如图 13 所示, 当对其进行加 热时, 整个结构就会折叠成预期的形状。该团队以此 为基础在折纸上增加了磁铁, 设计了一个多场驱动的 多功能运动平台, 如图 14a 所示, 该平台的加热区域 可对热缩型材料进行加热, 实现折纸的折叠运动, 然 后通过磁场实现折叠状态折纸的滚动, 并将该结构用 于模拟人体环境的药物抓取和释放 ${ }^{[47]}$, 如图 $14 b$ 所示。

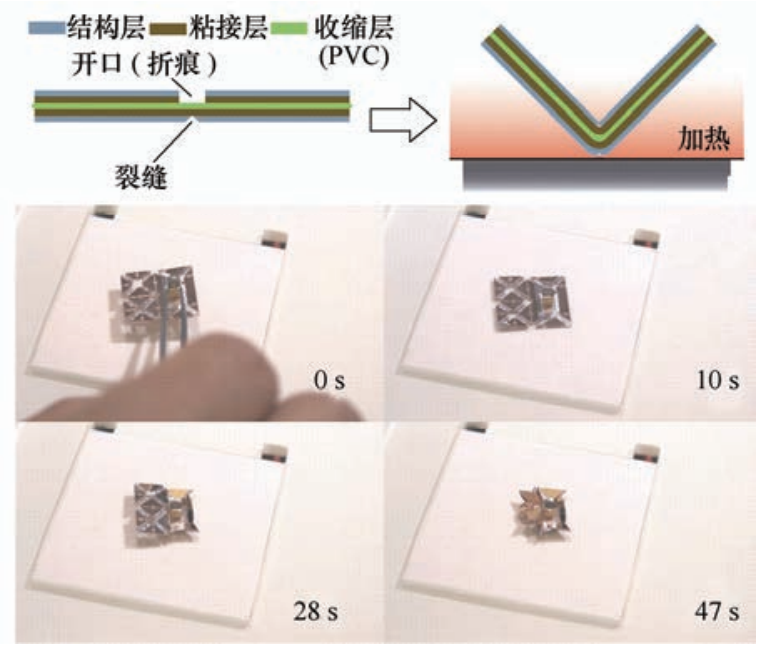

图 13 热缩型材料驱动的折纸

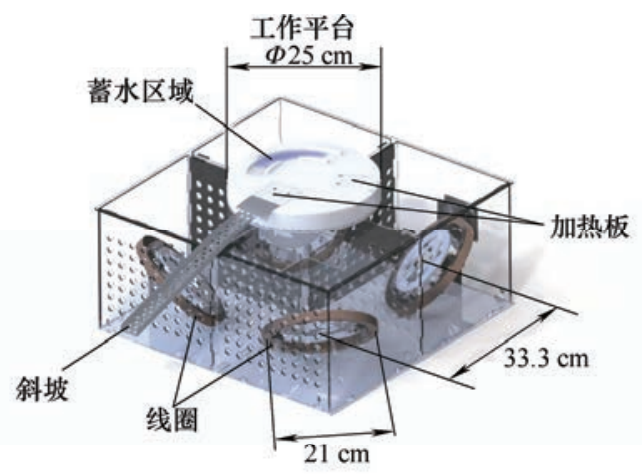

(a) 多场驱动工作平台

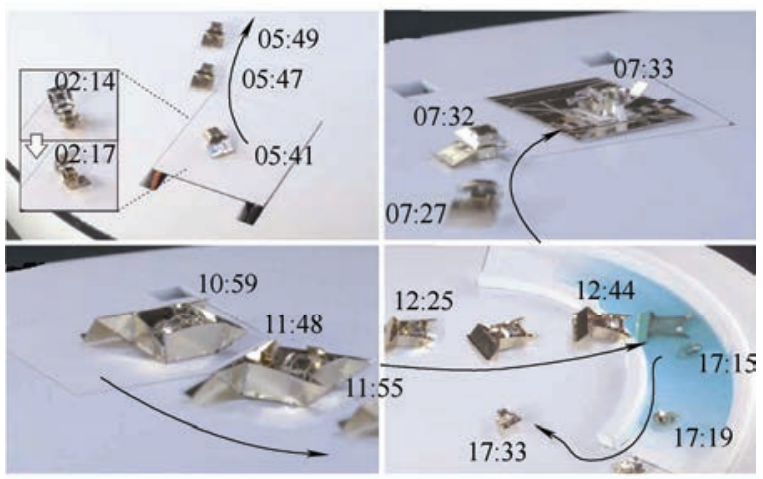

(b) 药物抓取机器人在模拟人体环境中的工作过程

图 14 温度和磁场同时作用的药物抓取释放机器人 


\section{2 电活性材料驱动}

电活性材料是一类在电场激励下可以产生大幅 度尺寸或形状变化的新型柔性功能材料, 与压电陶 瓷、形状记忆合金等传统功能材料相比, 电活性材 料具有变形大、响应迅速、功耗低、质量小、柔韧 性好等众多优势, 因此在折纸驱动方面也表现出巨 大的发展潜力。美国 BRUCE 等 ${ }^{[48]}$ 利用离子型水凝 胶电活性材料在电压作用下形状改变的特点研制了 一种弯曲变形驱动器, 如图 15 所示, 并通过该驱动 器实现了软管的折叠和展开运动。日本 OKUZAKI 等 ${ }^{[49]}$ 设计了一种基于导电薄膜的驱动器, 这种导电 薄膜在电场作用下可发生水分子的吸附和解吸, 导 致弹性模量发生变化, 从而可以实现反复拉伸和收 缩运动, 他们用这种材料制成管状折纸, 并为其装 上与地面接触的 “腿” 部, 最终实现了向前蠕动爬 行的运动功能, 如图 16 所示。

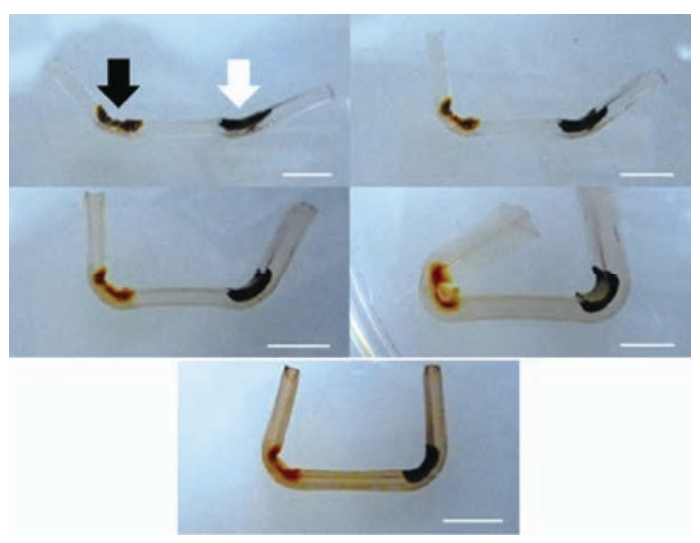

图 15 离子型水凝胶驱动的可折叠软管
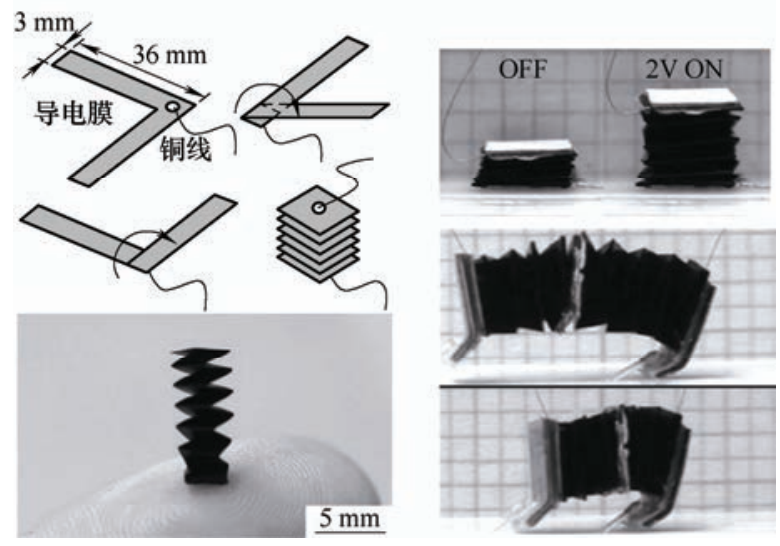

图 16 导电薄膜驱动的管状折纸爬行器

除了已有报导将电活性材料应用于折纸结构驱 动外, 还有一些新型电活性材料在电场作用下具备 直接或间接产生弯曲变形的能力, 同样有望用于折 纸的驱动。例如, 离子聚合物-金属复合材料(Ionic polymer-mental composite, IPMC)可以在 3 $5 \mathrm{~V}$ 电 压作用下内部阳离子携带部分溶剂分子向阴极迁
移, 引起阴极区域离子和溶剂聚集而膨胀, 产生弯 曲变形, 如图 17a 所示, 若将 IPMC 置于折纸的折 痕处, 便可在电压作用下实现折叠和展开。聚氯乙 烯凝胶(PVC gel)可以在数百伏电场的作用下产生 Maxwell 应力以及静电吸附效应, 在强大的吸附作 用下材料会朝着阳极一侧弯曲, 如图 17b 所示。 HIRAI 等 ${ }^{[50]}$ 研究了电极结构与 PVC gel 弯曲变形之 间的关系, 结果显示可达到接近 $180^{\circ}$ 的弯曲角度, 因此 PVC gel 也具有驱动折纸机构的潜在价值。介 电弹性体(Dielectric elastomer, DE) 可以在数千伏的 电场下产生面内扩张, 虽然不能直接产生弯曲变形, 但是可以通过一些结构设计转化为弯曲变形。哈尔 滨工业大学 ZHAO 等 ${ }^{[51]}$ 根据最小势能原理利用 DE 薄膜设计了一种弯曲变形驱动器, 如图 17c 所示, 它可实现由面内扩张变形向弯曲变形的转换, 该设 计也可以作为一种驱动折纸的方案。

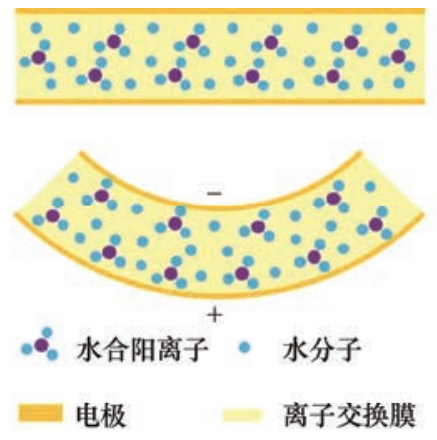

(a) IPMC 的弯曲变形

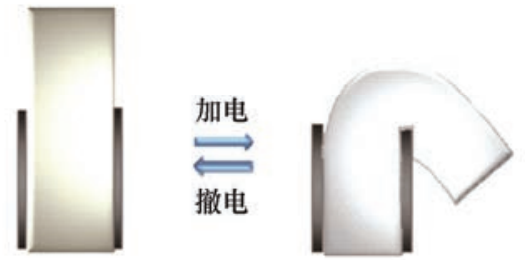

(b) PVC gel 的弯曲变形

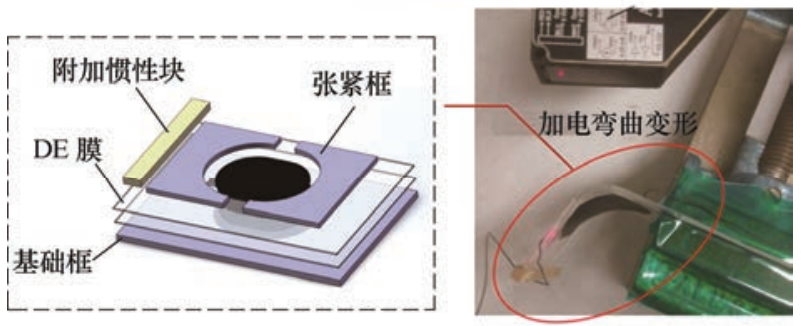

(c) 基于最小势能原理的 DE 可弯曲变形结构

图 17 具有驱动折纸潜力的电活性材料

\section{3 其他活性材料驱动}

除了热活性材料、电活性材料外, 活性材料还包 括化学活性、磁活性、光活性等材料, 它们均可以在 一定条件下发生弯曲变形, 从而驱动折纸。例如, BASSIK 等 ${ }^{[52]}$ 将具有生化活性的材料用作折纸的折 痕, 然后通过活性酶促发化学反应致使材料性质改 
变, 从而实现折纸的折叠与展开, 并据此设计了一种 微观抓取器, 如图 18a 所示。香港大学 KWAN 等 ${ }^{[53]}$ 研制了一种光驱动爬行机器人, 如图 18b 所示, 他们 在爬行机器人工作前先将其前后腿在乙醇中放置不 同长度时间而导致不同的变形, 使前后腿形成高度 差, 然后放入可见光照中, 在光作用下弯曲角度小的 腿与弯曲角度大的腿移动速度不一致, 最终使爬行机 器人在光照开关交替变化的条件下朝着光的方向运 动。宾夕法尼亚州立大学研究人员等将 30\%的钡铁 氧体颗粒混合到硅橡胶基体中研制出了一种磁活性弹 性体(Magnetically active elastomer, MAE), 这种 MAE 材料可以在磁场环境中产生弯曲变形, 他们以此设计 了一种驱动器, 并且将 MAE 贴片粘在一个立方体盒子 的四个侧面, 若将此盒子置于特定磁场中, 它就可以 把二维平面折叠成三维立方体, 如图 18c 所示 ${ }^{[54]}$ 。

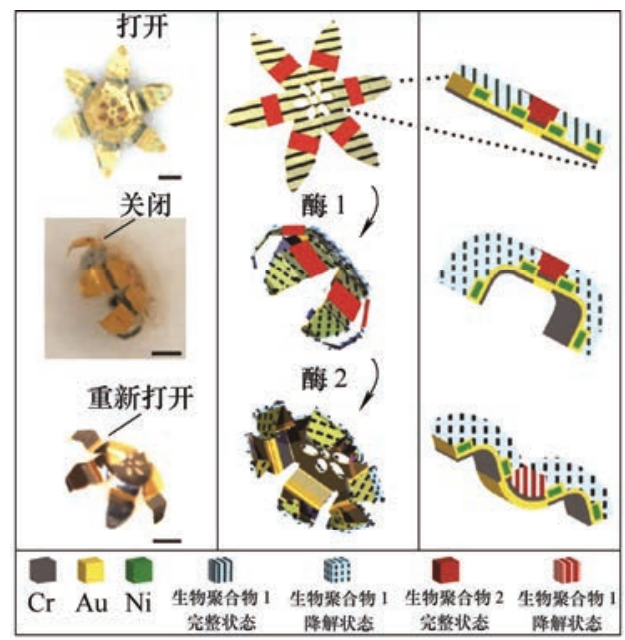

(a) 化学活性材料驱动的抓取器

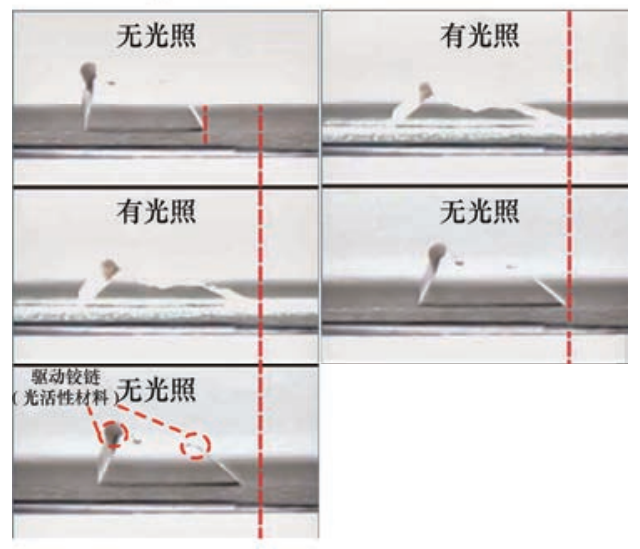

(b) 光驱动的爬行机器人

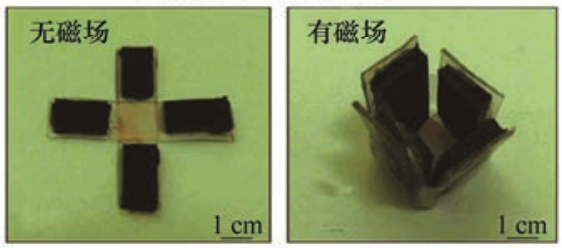

(c) 磁活性材料驱动的立方体折纸结构

图 18 化学活性、光活性、磁活性材料驱动的折纸

\section{4 活性材料驱动性能比较}

活性材料具有多种多样的变形形式，而基于活 性材料的折纸驱动结构具有结构简单、变形灵活等 特点, 将活性材料与折纸结构的结合可充分发挥它 们各自特有的优势，实现折纸由 “被折叠” 到 “自 折叠” 的转换，从而使得折纸的运动与控制更为 “智 能”。因而, 活性材料驱动是一种十分重要的折纸结 构驱动方法, 特别适用于一些小型折纸机构的驱动, 也将进一步扩大折纸结构的应用领域。

由于活性材料种类繁多，其性能之间差别也很 大，考虑到活性材料的应力和应变特性直接关系到 折纸的驱动性能, 图 19 给出了部分活性材料的应力 应变范围示意图 ${ }^{[55]}$ 。由图可见, 对于绝大多数活性 材料而言，一般很难同时具有应力和应变均较大的 特性, 这就需要在驱动结构设计时做到“有取有舍”, 即根据折纸结构所需的变形和驱动力范围选择恰当 的材料。

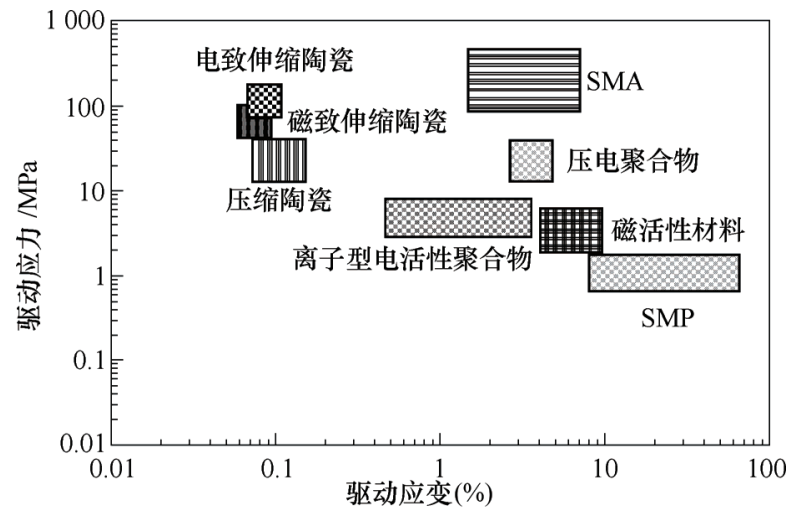

图 19 活性材料的应力应变范围示意图

此外，不同活性材料的驱动响应速度也有明显 差异, 例如, 部分电活性材料仅需要零点几秒就可 产生变形, 而一些热活性材料需要数分钟才能产生 变形，这显然会限制材料的应用场合。因此，在采 用活性材料对折纸结构驱动时必须考虑时间效率。

\section{4 结论与展望}

折纸结构具有很强的折展性能, 并且在实际应 用中具有制造装配简单等特点, 可以满足不少工程 领域中的减重以及结构形状或尺寸可变的需求，因 而近年来吸引了国内外众多学者的关注。为了促进 折纸技术的快速发展，本文从折纸结构的驱动层面 对其研究现状进行介绍与分析, 将现有的折纸驱动 方式分为气动驱动、机械式驱动、活性材料驱动三 大类, 为了清晰比较起见, 表 1 归纳了这三大类驱 动方法的优缺点以及适用场合。 
表 1 常见折纸结构驱动方法比较

\begin{tabular}{|c|c|c|c|c|}
\hline & 驱动方法 & 优势 & 劣势 & 适用场合 \\
\hline & 气动驱动 & $\begin{array}{l}\text { 驱动力大, 折叠范围大, 容易实现折纸 } \\
\text { 的拉伸和收缩运动, 可通过结构设计实现 } \\
\text { 管状折纸的弯曲运动 }\end{array}$ & $\begin{array}{l}\text { 需要气泵等辅助设备, 对于难以形 } \\
\text { 成封闭腔体的折纸或不具备气沝设备 } \\
\text { 的特殊场合无法使用 }\end{array}$ & $\begin{array}{l}\text { 具有封闭腔体的折纸结构且具 } \\
\text { 有气压控制设备 }\end{array}$ \\
\hline & 机械式驱动 & $\begin{array}{l}\text { 驱动力和运动范围大, 能够对结构进行 } \\
\text { 较为精准的控制, 可通过结构设计可以完 } \\
\text { 成复杂运动 }\end{array}$ & $\begin{array}{l}\text { 需要绳索、齿轮、铰链、弹簧等机 } \\
\text { 械零部件, 结构复杂, 不利于小型结 } \\
\text { 构的制造装配和减重 }\end{array}$ & 对驱动力要求大的大型折纸结 \\
\hline \multirow{4}{*}{$\begin{array}{l}\text { 活 } \\
\text { 性 } \\
\text { 材 } \\
\text { 料 } \\
\text { 驱 } \\
\text { 动 }\end{array}$} & 热活性材料 & $\begin{array}{l}\text { 结构简单, 驱动力和变形范围较大, 可 } \\
\text { 对折纸的每一处折痕进行独立控制 }\end{array}$ & $\begin{array}{l}\text { 对温度场进行快速准确地控制比较 } \\
\text { 困难, 加热和散热过程存在效率问题 }\end{array}$ & $\begin{array}{l}\text { 应用对象可以承受高温环境, } \\
\text { 工作环境便于加热和散热 }\end{array}$ \\
\hline & 电活性材料 & $\begin{array}{l}\text { 结构简单, 响应迅速, 变形范围大, 工 } \\
\text { 作所需条件易于实现 }\end{array}$ & 驱动力小, 部分材料需要高电压 & $\begin{array}{l}\text { 对驱动力要求不高的小型折纸 } \\
\text { 结构 }\end{array}$ \\
\hline & \multirow{2}{*}{$\begin{array}{l}\text { 化学、磁、光等活 } \\
\text { 性材料 }\end{array}$} & 结构简单, 驱动力和变形范围较大 & $\begin{array}{l}\text { 对工作环境要求苛刻, 通用性不强, } \\
\text { 部分材料响应较慢 }\end{array}$ & $\begin{array}{l}\text { 工作环境具有化学溶液、磁场、 } \\
\text { 光照等条件 }\end{array}$ \\
\hline & & $\begin{array}{l}\text { 结构简单, 体积小, 重量小, 可供选择 } \\
\text { 的材料种类多 }\end{array}$ & $\begin{array}{l}\text { 驱动力小, 存在效率问题, 对工作 } \\
\text { 环境有一定要求 }\end{array}$ & $\begin{array}{l}\text { 对驱动力要求不高的微小型折 } \\
\text { 纸结构 }\end{array}$ \\
\hline
\end{tabular}

显然, 每一种驱动方法都有其特点, 而驱动方 式的优劣直接决定着折纸结构优势的发挥。因此, 折纸的驱动模式设计并非一个简单的单向设计问 题, 而是一个复杂的交互过程, 即在选择驱动方法 的同时必须考虑折纸的运动特点, 而在折纸结构的 设计阶段也必须同时考虑驱动方法, 只有综合考虑 结构设计和驱动方法才能发挥出折纸结构在工程中 应用的优势。

折纸结构是近年来迅速发展的一个新方向, 在 航天工程、生物医学工程、机器人以及其他一些需 要结构能够折叠与展开的多个领域均具有重要的应 用前景。总体来看, 折纸技术在工程中的应用目前 还处于探索阶段, 除了需要深入研究折纸理论、开 发新型折纸结构之外, 折纸结构的驱动技术也是一 个重要的研究领域。

对于复杂的大型折纸结构, 气动驱动和机械式 驱动是比较好的选择, 而如何使得折纸结构实现更 为精准、智能和复杂多样的运动功能将会是未来研 究的热点问题, 这也必将涉及结构、材料、控制、

人工智能等多个领域。

在轻小型折纸结构中, 应用智能活性材料对折 纸结构进行驱动将是一种必然趋势。分析现有研究 活性材料在折纸结构驱动中的应用可以发现, 要想 充分发挥折纸结构的优势, 未来将活性材料应用于 折纸结构驱动至少存在以下问题亟需解决, 它也是 该技术未来的发展方向。

(1) 在轻小型折纸结构的驱动中, 人们希望驱 动材料同时具有应力和应变均较大的特性, 但目前 同时满足这两个要求的活性材料还寒胗无几。因此, 开发性能优异的新型活性材料用于轻小型折纸结构 的驱动是一个重要的发展方向。
（2）现有的活性材料种类繁多，不同的活性材 料具有不同的特征。若要对不同折纸结构实现有效 驱动, 就需要对不同折纸结构进行必要的几何和力 学分析, 与此同时分析不同活性材料的驱动性能, 这样才能充分利用及发挥现有活性材料的优势并应 用于折纸结构中。因此，对现有不同活性材料的深 入研究及其在轻小型折纸结构中的应用研究也将会 是一个发展方向。

（3）对于轻小型折纸结构来讲, 质量轻是一个 关键性能指标, 但是为了实现折纸的折展必然引入 驱动材料或驱动结构的附加质量, 显然这对轻小型 折纸结构是不希望的。因此, 利用智能材料实现轻 小型折纸结构的驱动材料和折纸材料一体化, 即同 一种材料同时发挥折纸片和驱动的作用, 这种设计 将会更好地发挥折纸的优势, 使得结构更加紧凑, 将会成为另一个发展方向。

（4）在许多实际应用场合，人们不仅希望折纸 结构能够完成大尺度的形状变化, 而且希望该折纸 结构能够完成在一定空间的运动, 也就是说将不同 功能集成在一个折纸结构中, 类似于图 14 中的驱动 方法, 折纸上同时附着热活性材料和磁铁材料, 热 活性材料完成折纸的折叠变形, 外界磁场用于完成 折叠状态结构的滚动运动, 这样的设计方法可使折 纸完成更为复杂的动作, 这也将是折纸驱动技术未 来发展的一大趋势。

\section{参 考 文 献}

[1] LANG R J. The science of origami[J]. Physics World, 2007, 20(2): 30-31.

[2] TACHI T. Origamizing polyhedral surfaces[J]. IEEE Transactions on Visualization \& Computer Graphics, 
2010, 16(2): 298-311.

[3] DEMAINE E D. Folding and unfolding linkages, paper, and polyhedra[M]. Berlin Heidelberg: Springer, 2000.

[4] MUELLER S, KRUCK B , BAUDISCH P. Laser Origami: Laser-cutting 3D objects[C]// Proceedings of the SIGCHI Conference on Human Factors in Computing Systems, New York: ACM, 2013: 2585-2592.

[5] ZIRBEL S A, WILSON M E, MAGLEBY S P, et al. An origami-inspired self-deployable array[C]// ASME Smart Materials, Adaptive Structures and Intelligent Systems, New York: ASME, 2013: V001T01A026.

[6] RANDALL C L, GULTEPE E, GRACIAS D H. Selffolding devices and materials for biomedical applications[J]. Trends in Biotechnology, 2012, 30(3): 138-146.

[7] FERNANDES R, GRACIAS D H. Self-folding polymeric containers for encapsulation and delivery of drugs[J]. Advanced Drug Delivery Reviews, 2012, 64(14) : 1579-1589.

[8] WANG Yanhu, GE Lei, WANG Panpan, et al. A three-dimensional origami-based immuno-biofuel cell for self-powered, low-cost, and sensitive point-of-care testing[J]. Chemical Communications, 2014, 50(16): 1947-1949.

[9] EDMONDSON B, BOWEN L, GRAMES C, et al. Oriceps: Origami-inspired forceps[C]// ASME 2013 Conference on Smart Materials, Adaptive Structures and Intelligent Systems, American Society of Mechanical Engineers, New York: ASME, 2013: V001T01A027.

[10] KURIBAYASHI K, TSUCHIYA K, YOU Z, et al. Self-deployable origami, stent grafts as a biomedical application of Ni-rich TiNi shape memory alloy foil[J]. Materials Science \& Engineering A, 2006, 419(1): 131-137.

[11] YOON C, XIAO R, PARK J, et al. Functional stimuli responsive hydrogel devices by self-folding[J]. Smart Materials and Structures, 2014, 23(9): 094008.

[12] LEE D Y, KIM J S, KIM S R, et al. The deformable wheel robot using magic-ball origami structure[C]// ASME International Design Engineering Technical Conferences and Computers and Information in Engineering Conference, New York: ASME, 2013: V06BT07A040.

[13] WHITE P J, LATSCHA S, SCHLAEFER S, et al. Dielectric elastomer bender actuator applied to modular robotics[C]// IEEE International Conference on Intelligent Robots and Systems, Piscataway: IEEE, 2011: 408-413.
[14] HOOVER A M, FEARING R S. Fast scale prototyping for folded millirobots[C]// IEEE International Conference on Robotics and Automation, Piscataway: IEEE, 2008: 886-892.

[15] LEE J Y, KANG B B. Development of a multi-functional soft robot (snUMaX) and performance in robosoft grand challenge[J]. Frontiers in Robotics and AI, 2016, 3: UNSP63.

[16] SNYDER M P, SANDERS B, EASTEP F E, et al. Vibration and flutter characteristics of a folding wing[J]. Journal of Aircraft, 2009, 46(3): 791-799.

[17] BUNGET G, SEELECKE S. BATMAV: A 2-DOF bio-inspired flapping flight platform[J]. Proceedings of SPIE the International Society for Optical Engineering, 2010, 7643(2): 379-380.

[18] POUNDS P E. Paper plane: Towards disposable low-cost folded cellulose-substrate UAVs[C]// Proceedings of Australian Robotics and Automation Association , Sydney: ARAA, 2012: 143-151.

[19] MA Jiayao, YOU Zhong. The origami crash box[J]. Origami, 2011(5): 277-290.

[20] MA Jiayao, YOU Zhong. A novel origami crash box with varying proles[C]// ASME 2013 International Design Engineering Technical Conferences and Computers and Information in Engineering Conference, New York: ASME, 2013: V06BT07A048.

[21] ZHOU Caihua, WANG Bo, MA Jiayao, et al. Dynamic axial crushing of origami crash boxes[J]. International Journal of Mechanical Sciences, 2016, 118: 1-12.

[22] TOLMAN S S, DELIMONT I L, HOWELL L L, et al. Material selection for elastic energy absorption in origami-inspired compliant corrugations[J]. Smart Materials and Structures, 2014, 23(9): 094010.

[23] WU Weina, YOU Zhong. A solution for folding rigid tall shopping bags[J]. Proceedings of the Royal Society A: Mathematical, Physical and Engineering Sciences, 2011, 467(2133): 2561-2574.

[24] CHENG Qianqian, SONG Zemin, MA Teng, et al. Folding paper-based lithium-ion batteries for higher areal energy densities[J]. Nano Letters, 2013, 13(10) : 4969-4974.

[25] SONG Zemin, MA Teng, TANG Rui, et al. Origami lithium-Ion batteries[J]. Nature Communications, 2014(5): 1810-1821.

[26] NAM I, KIM G P, PARK S, et al. All-solid-state, origa- 
mi-type foldable super capacitor chips with integrated series circuit analogues[J]. Energy and Environmental Science, 2014, 7(3): 1095-1102.

[27] MIYASHITA S, MEEKER L, TOLLEY M T, et al. Self-folding miniature elastic electric devices[J]. Smart Materials and Structures, 2014， 23(9): 094005.

[28] OCAMPO J, ZANARDI M, VACCARO P O, et al. Characterization of GaAs-based micro-origami mirrors by optical actuation[J]. Microelectronic Engineering, 2004, 73(1): 429-434.

[29] EARLY J T, HYDE R, BARON R L. Twenty-meter space telescope based on diffractive Fresnel lens[C]// SPIE 48th Annual Meeting Cardiff: SPIE, 2004: 148-156.

[30] THRALL A, QUAGLIA C. Accordion shelters: A historical review of origami-like deployable shelters developed by the US military[J]. Engineering Structures, 2014(59): 686-692.

[31] MARTIN F, THRALL A. Honeycomb core sandwich panels for origami-inspired deployable shelters: Multiobjective optimization for minimum weight and maximum energy efficiency[J]. Engineering Structures, 2014(69): 158-167.

[32] QUAGLIA C, BALLARD Z, THRALL A. Parametric modeling of an air-liftable origami-inspired deployable shelter with a novel erection strategy[J]. Mobile and Rapidly Assembled Structures IV， 2014(136): 23-33.

[33] FUCHI K, DIAZ A R, ROTHWELL E J, et al. An origami tunable metamaterial[J]. Journal of Applied Physics, 2012, 111(8): 2075-2084.

[34] SCHENK M, GUEST S D. Geometry of Miura-folded metamaterials[J]. PNAS, 2013, 110(9): 3276-3281.

[35] MARTINEZ R V, FISH C R, CHEN X, et al. Elastomeric origami: Programmable paper-elastomer composites as pneumatic actuators[J]. Advanced Function Material, 2012(22): 1376-1384.

[36] FANG Hongbin, ZHANG Yetong, WANG K W. Origami-based earthworm-like locomotion robots[J]. Bioinspiration and Biomimetics, 2017, 12: 065003.

[37] LI S, VOGT D M, RUS D, et al. Fluid-driven origami-inspired artificial muscles[J]. Proceedings of the National Academy of Sciences of the United States of America, 2017, 114(50): 13132-13137.

[38] KIM S J, LEE D Y, JUNG G P, et al. An origamiinspired, self-locking robotic arm that can be folded flat[J]. Science Robotics, 2018, 3(16): eaar2915.
[39] ZHANG Ketao, QIU Chen, DAI Jiansheng. An origami parallel structure integrated deployable continuum robot[C]// ASME 2015 International Design Engineering Technical Conferences and Computers and Information in Engineering Conference, New York: ASME, 2015: V05BT08A032.

[40] JEONG D, LEE K. Design and analysis of an origamibased three-finger manipulator[J]. Robotica， 2018，36: 261-274.

[41] KAMRAVA S, MOUSANEZHAD D, FELTON S M, et al. Programmable Origami strings[J]. Advanced Materials Technologies, 2018: 1700276.

[42] TOLLEY M T, FELTON S M, MIYASHITA S, et al. Self-folding origami: Shape memory composites activated by uniform heating[J]. Smart Materials and Structures, 2014, 23(9): 094006.

[43] FELTON S, TOLLEY M, DEMAINE E, et al. A method for building self-folding machines[J]. Science, 2014, 345(6197): 644-646.

[44] PAIK J K, AN B, RUS D, et al. Robotic origamis: Self-morphing modular robot[J]. ICMC， 2012: 206919.

[45] ONAL C D, WOOD R J, RUS D. An origami-inspired approach to worm robots[J]. IEEE/ASME Trans. Mechatron, 2013, 18: 430-438.

[46] MIYASHITA S, GUITRON S, LUDERSDORFER M, et al. An untethered miniature origami robot that self-folds, walks, swims, and degrades[C]// IEEE 2015 International Conference on Robotics and Automation, Piscataway: IEEE, 2015: 1490-1496.

[47] MIYASHITA S, GUITRON S, LI S, et al. Robotic metamorphosis by origami exoskeletons[J]. Science Robotics, 2017, 2: eaao4369.

[48] BRUCE P L, AMEYA N, RANDALL W. Effect of metal ion type on the movement of hydrogel actuator based On catechol-metal ion coordination chemistry[J]. Sensors and Actuators B, 2016 (227): 248-254.

[49] OKUZAKI H, SAIDO T, SUZUKI H, et al. A biomorphic origami actuator fabricated by folding a conducting paper[J]. Journal of Physics: Conference Series, 2008, 127: 012001

[50] HIRAI T, KOBAYASHI S, HIRAI M, et al. Bending induced by creeping of plasticized poly(vinyl chloride) gel[J]. Proceedings of SPIE-The International Society for Optical Engineering, 2004, 5385: 433-441.

[51] ZHAO Jianwen, NIU Junyang, MCCOUL D, et al. 
Phenomena of nonlinear oscillation and special resonance of a dielectric elastomer minimum energy structure rotary joint[J]. Applied Physics Letters, 2015, 106(13): 133504.

[52] BASSIK N, BRAFMAN A, ZARAFSHAR A M, et al. Enzymatically triggered actuation of miniaturized tools[J]. Journal of the American Chemical Society, 2010, 132(46): 16314-16317.

[53] KWAN K W, LI S J, HAU N Y, et al. Light-stimulated actuators based on nickel hydroxide-oxyhydroxide[J]. Science Robotics, 2018， 3: eaat4051.

[54] AHMED S, LAUFF C, CRIVARO A, et al. Multi-field responsive origami structures: Preliminary modeling and
experiments[C]// ASME 2013 International Design Engineering Technical Conferences and Computers and Information in Engineering Conference, New York: ASME, 2013, DETC-12405: V06BT07A028.

[55] LAGOUDAS D C. Shape memory alloys: Modeling and engineering applications[M]. Berlin Heidelberg: Springer, 2008.

作者简介: 胡楠, 男, 1992 年出生, 博士研究生。主要研究方向为折纸 结构的驱动技术。

E-mail: nanh_2016@126.com

陈花玲(通信作者), 女, 1954 年出生, 博士, 教授, 博士研究生导师。 主要研究方向为智能材料与结构控制, 噪声与振动分析。

E-mail: hlchen@mail.xjtu.edu.cn 